Research Article

\title{
Generalized Weighted Statistical Convergence for Double Sequences of Fuzzy Numbers and Associated Korovkin-Type Approximation Theorem
}

\begin{abstract}
Abdullah Alotaibi
Operator Theory and Applications Research Group, Department of Mathematics, Faculty of Science, King Abdulaziz University, P.O. Box 80203, Jeddah 21589, Saudi Arabia
\end{abstract}

Correspondence should be addressed to Abdullah Alotaibi; mathker11@hotmail.com

Received 7 April 2020; Accepted 7 May 2020; Published 8 June 2020

Guest Editor: Acu Ana Maria

Copyright (C) 2020 Abdullah Alotaibi. This is an open access article distributed under the Creative Commons Attribution License, which permits unrestricted use, distribution, and reproduction in any medium, provided the original work is properly cited.

\begin{abstract}
We define the notions of weighted $(\lambda, \mu)$-statistical convergence of order $\left(\gamma_{1}, \gamma_{2}\right)$ and strongly weighted $(\lambda, \mu)$-summability of $\left(\gamma_{1}, \gamma_{2}\right)$ for fuzzy double sequences, where $0<\gamma_{1}, \gamma_{2} \leq 1$. We establish an inclusion result and a theorem presenting a connection between these concepts. Moreover, we apply our new concept of weighted $(\lambda, \mu)$-statistical convergence of order $\left(\gamma_{1}, \gamma_{2}\right)$ to prove Korovkin-type approximation theorem for functions of two variables in a fuzzy sense. Finally, an illustrative example is provided with the help of $q$-analogue of fuzzy Bernstein operators for bivariate functions which shows the significance of our approximation theorem.
\end{abstract}

\section{Introduction and Preliminaries}

The notion of weighted statistical convergence for sequences of real numbers has been studied by Karakaya and Chishti [1] as a generalization of the concept of statistical convergence which is related to the idea of asymptotic density (or natural density) of a subset of $\mathbb{N}$, the set of natural numbers, according to Fast [2]. The weighted statistical convergence was further improved by Mursaleen et al. [3] and later generalized by Belen and Mohiuddine [4] with a view of nondecreasing sequence of positive numbers. Ghosal [5] added constraints to these ideas. For some other related work, we refer the interested reader to [6-10]. Recently, Çolak [11] defined the notion of statistical convergence of order $\alpha(0<\alpha \leq 1)$ and strong $p$-Cesàro summability of order $\alpha$ while the order of statistical was also given in [12], and it reduces to strong $p$-Cesàro summability for $\alpha=1$ according to Connor [13].

The idea convergence for double sequence $y=\left(y_{k l}\right)$ was presented by Pringsheim [14]; that is, $\left(y_{k l}\right)$ is convergent to $\xi$ in the Pringsheim sense if for every $\varepsilon>0$, there is $N \in \mathbb{N}$ such that $\left|y_{k l}-\xi\right|<\varepsilon$ whenever $k, l>N$. The idea of statistical convergence for $y=\left(y_{k l}\right)_{(k, l) \in \mathbb{N} \times \mathbb{N}}$ was introduced by
Mursaleen and Edely [15] as follows: $\left(y_{k l}\right)$ is statistically convergent to $\xi$ if for every $\varepsilon>0$,

$$
\lim _{k_{1}, l_{1} \rightarrow \infty} \frac{1}{k_{1} l_{1}}\left\{(k, l), k \leq k_{1}, l \leq l_{1}:\left|y_{k l}-\xi\right| \geq \epsilon\right\}=0,
$$

where the limit is taken in Pringsheim's sense while the order of notion was studied in [16]. This idea was further generalized by Mursaleen et al. [17] and called it $(\lambda, \mu)$-statistical convergence, and for single sequence in [18] and its weighted variant were presented by Cinar and Et [19], which were later on studied by various authors in various setups [20-22]. Throughout this paper, limit of the double sequences means limit in Pringsheim's sense.

In the very recent past, for sequence of fuzzy numbers, Mohiuddine et al. [23] weighted statistical convergence and strong weighted summability by using the idea of difference operator and established a connection between these notions while under certain conditions, these notions reduce to statistical convergence and strong $p$-Cesáro summability, respectively, in [24, 25]. Savas and Mursaleen [26] defined statistical convergence and statistically Cauchy for fuzzy 
double sequences and obtained that these concepts are equivalent. The statistical analogue of pointwise and uniformly convergent double sequences for fuzzy-valued functions were discussed by Mohiuddine et al. [21]. These concepts were studied and extended by several authors; for instance, see [27-31].

Recall that, in [32], a fuzzy number is a fuzzy set on the real axis; that is, $u: \mathbb{R} \longrightarrow[0,1]$ which is normal, fuzzy convex, upper semicontinuous and the closure of the set $\{y \in$ $\mathbb{R}: u(y)>0\}$ is compact. By using the symbol $\mathscr{L}(\mathbb{R})$, we denote the set of all fuzzy numbers on $\mathbb{R}$. $\alpha$-Level set $[u]_{\alpha}$ of $u$ is given as follows:

$$
[u]_{a}= \begin{cases}\{y \in \mathbb{R}: u(y) \geq \alpha\} & \text { if } 0<\alpha \leq 1, \\ \overline{\{y \in \mathbb{R}: u(y)>0\}} & \text { if } \alpha=0 .\end{cases}
$$

For each $\alpha \in[0,1],[u]_{\alpha}$ is closed and bounded and nonempty interval for each $\alpha \in[0,1]$ defined by $[u]_{\alpha}=\left[u_{\alpha}^{-}, u_{\alpha}^{+}\right]$, where $u_{\alpha}^{-} \leq u_{\alpha}^{+}$and $u_{\alpha}^{-}, u_{\alpha}^{+} \in \mathbb{R}$. For any $u_{1}, u_{2} \in \mathscr{L}(\mathbb{R})$, the partial ordering is defined by

$$
\begin{aligned}
& u_{1} \preceq u_{2} \Leftrightarrow u_{1 \alpha}^{-} \leq u_{2 \alpha}^{-}, \\
& u_{1 \alpha}^{+} \leq u_{2 \alpha}^{+},
\end{aligned}
$$

for all $0 \leq \alpha \leq 1$. For any $u_{1}, u_{2} \in \mathscr{L}(\mathbb{R})$ and $\alpha \in[0,1]$, define the following:

$$
\begin{aligned}
{\left[u_{1} \oplus u_{2}\right]_{\alpha} } & =\left[u_{1}\right]_{\alpha} \oplus\left[u_{2}\right]_{\alpha}, \\
{\left[\mu \odot \mu_{1}\right]_{\alpha} } & =\mu\left[u_{1}\right]_{\alpha} \quad(\mu \in \mathbb{R}) .
\end{aligned}
$$

By means of the Hausdorff metric $D$, the metric $d$ : $\mathscr{L}(\mathbb{R}) \times \mathscr{L}(\mathbb{R}) \longrightarrow \mathbb{R}_{+}$is defined by

$$
\begin{aligned}
d\left(u_{1}, u_{2}\right) & =\sup _{\alpha \in[0,1]} D\left(\left[u_{1}\right]_{\alpha},\left[u_{2}\right]_{\alpha}\right) \\
& =\sup _{\alpha \in[0,1]} \max \left\{\left|u_{1 \alpha}^{-}-u_{2 \alpha}^{-}\right|,\left|u_{1 \alpha}^{+}-u_{2 \alpha}^{+}\right|\right\}
\end{aligned}
$$

It is clear from [33] that $(\mathscr{L}(\mathbb{R}), d)$ is a complete metric space.

Suppose $g_{1}, g_{2}:[a, b] \times[a, b] \longrightarrow \mathscr{L}(\mathbb{R})$ are two fuzzy number-valued functions. Then, the distance between $g_{1}$ and $g_{2}$ is defined by

$$
\begin{aligned}
d^{*}\left(g_{1}, g_{2}\right)= & \sup _{y \in[a, b] \times[a, b] \alpha \in[0,1]} \sup _{\max } \\
& \cdot\left\{\left|g_{1 \alpha}^{-}-g_{2 \alpha}^{-}\right|,\left|g_{1 \alpha}^{+}-g_{2 \alpha}^{+}\right|\right\} .
\end{aligned}
$$

\section{Generalized Weighted Statistical Convergence of Order $\left(\gamma_{1}, \gamma_{2}\right)$}

Suppose two nondecreasing sequence $\lambda=\left(\lambda_{k}\right)$ and $\mu=\left(\mu_{l}\right)$ of positive integers such that both $\lambda$ and $\mu$ tending to infinity; that is, $\lambda_{k} \longrightarrow \infty$ and $\mu_{l} \longrightarrow \infty$ as $k \longrightarrow \infty$ and $l \longrightarrow \infty$, respectively, such that

$$
\begin{array}{ll}
\lambda_{k+1} \leq \lambda_{k}+1, & \lambda_{k}=1, \\
\mu_{l+1} \leq \mu_{l}+1, & \mu_{l}=1 .
\end{array}
$$

Consider two sequences $u=\left(u_{m}\right)$ and $v=\left(v_{n}\right)$ of nonnegative numbers such that $\liminf _{m} u_{m}>0, \liminf _{n} v_{n}>0$ and

$$
\begin{array}{r}
U_{\lambda_{k}}=\sum_{m \in\left[k-\lambda_{k}+1, k\right]} u_{m} \longrightarrow \infty(k \longrightarrow \infty), \\
V_{\mu 1} \sum_{n \in[l-\mu l+1, l]} v_{n} \longrightarrow \infty(l \longrightarrow \infty) .
\end{array}
$$

Assume that $M \subseteq \mathbb{N} \times \mathbb{N}$ and let $0<\gamma_{1}, \gamma_{2} \leq 1$. We define the weighted $(\lambda, \mu)$ density of order $\left(\gamma_{1}, \gamma_{2}\right)$, denoted by $\delta_{2, \gamma_{1}, \gamma_{2}}^{\bar{N}(\lambda, \mu)}$, of $M$ by

$$
\delta_{2, \gamma 1, \gamma 2}^{\bar{N}(\lambda, \mu)}(M)=\lim _{k, l \rightarrow \infty} \frac{\left|A_{U_{\lambda_{k}}, V_{\mu_{l}}}\right|}{U_{\lambda k}^{\gamma 1} V_{\mu l}^{\gamma 2}},
$$

provided the limit in the last relation (9) exists, where

$$
A_{U_{\lambda_{k}}, V_{\mu l}}=\left\{m \leq U_{\lambda_{k}}, n \leq V_{\mu l}:(m, n) \in M\right\} .
$$

Definition 1. A fuzzy double sequence $\left(s_{m n}\right)$ is said to be weighted $(\lambda, \mu)$-statistically convergent of order $\left(\gamma_{1}, \gamma_{2}\right)$, where $0<\gamma_{1}, \gamma_{2} \leq 1$; in short, we shall write $S_{2, \gamma_{1}, \gamma_{2}}^{\bar{N}(\lambda, \mu)}$-convergent, to a fuzzy number $s_{0}$ if for every $\varepsilon>0$, the set

$$
B=\left\{(m, n) \in \mathbb{N} \times \mathbb{N}:: u_{m} v_{n} d\left(s_{m n}, s_{0}\right) \geq \epsilon\right\}
$$

has $\delta_{2, \gamma_{1}, \gamma_{2}}^{\bar{N}(\lambda, \mu)}$ density zero; that is,

$$
S_{2, \gamma 1, \gamma^{2}}^{\bar{N}(\lambda, \mu)}(B)=0
$$

Equivalently, we can write

$$
\begin{aligned}
& \lim _{k, l \rightarrow \infty} \frac{1}{U_{\lambda k}^{\gamma_{1}} V_{\mu l}^{\gamma_{2}}} \mid\left\{(m, n), m \leq U_{\lambda_{k}}, n\right. \\
& \left.\leq V_{\mu l}: u_{m} v_{n} d\left(s_{m n}, s_{0}\right) \geq \epsilon\right\} \mid=0 .
\end{aligned}
$$

We denote this convergence by $s_{m n} \stackrel{S_{2, \gamma_{1}, \gamma_{2}}^{\bar{N}(\lambda, \mu)}}{\longrightarrow} s_{0}$ or $S_{2, \gamma_{1}, \gamma_{2}}^{\bar{N}(\lambda, \mu)}-$ $\lim s_{m n}=s_{0}$. We denote the set of all $S_{2, \gamma_{1}, \gamma_{2}}^{\bar{N}(\lambda, \mu)}$-convergence by $S_{2, \gamma_{1}, \gamma_{2}}^{\bar{N}}(\lambda, \mu)$

The choice of $\gamma_{1}=\gamma_{2}=1$ in the notion of $S_{2, \gamma_{1}, \gamma_{2}}^{\bar{N}(\lambda, \mu)}$-convergence gives weighted $(\lambda, \mu)$-statistically convergence for fuzzy double sequences. In addition, if we choose $\lambda_{k}=k$, $\mu_{l}=l, u_{m}=1$, and $v_{n}=1$ for all $m, n$, then $S_{2, \gamma_{1}, \gamma_{2}}^{\bar{N}(\lambda, \mu)}$-convergence gives the notion of $(\lambda, \mu)$-statistically convergence in a fuzzy sense [26].

For $0<\gamma_{1}, \gamma_{2} \leq 1$, the notion of weighted $(\lambda, \mu)$-statistically convergent of order $\left(\gamma_{1}, \gamma_{2}\right)$ is well but not well defined 
for $\gamma_{1}, \gamma_{2}>1$. To show this assertion, we consider the following example.

Example 1. Assume that $\gamma_{1}, \gamma_{2}>1$, and define the fuzzy double sequence $\left(s_{m n}\right)$ by

$$
s_{m n}=\left\{\begin{array}{lll}
\overline{1} & \text { if } m+n & \text { even } \\
\overline{0} & \text { if } m+n & \text { odd }
\end{array}\right.
$$

Let $\varepsilon>0$ be given, and let $u_{m}=1$ and $v_{n}=1 \forall m, n \in \mathbb{N}$. Then,

$$
\begin{aligned}
& \lim _{k, l \rightarrow \infty} \frac{1}{U_{\lambda_{k}}^{\gamma 1} V_{\mu l}^{\gamma 2}}\left|\left\{(m, n), m \leq U_{\lambda_{k}}, n \leq V_{\mu l}: u_{m} v_{n} d\left(s_{m n}, \overline{1}\right) \geq \epsilon\right\}\right| \\
& \quad \leq \lim _{k, l \rightarrow \infty} \frac{\lambda_{k} \mu_{l}}{2 \lambda_{k}^{\gamma 1} \mu l^{\gamma_{2}}}=0 .
\end{aligned}
$$

On the other hand,

$$
\begin{aligned}
& \lim _{k, l \rightarrow \infty} \frac{1}{U_{\lambda_{k}}^{\gamma 1} V_{\mu l}^{\gamma 2}}\left|\left\{(m, n), m \leq U_{\lambda_{k}}, n \leq V_{\mu l}: u_{m} v_{n} d\left(s_{m n}, \overline{0}\right) \geq \epsilon\right\}\right| \\
& \quad \leq \lim _{k, l \rightarrow \infty} \frac{\lambda_{k} \mu_{l}}{2 \lambda_{k}^{\gamma 1} \mu l^{\gamma_{2}}}=0 .
\end{aligned}
$$

We conclude from (15) and (16) that

$$
\begin{aligned}
& S_{2, \gamma 1, \gamma^{2}}^{\bar{N}(\lambda, \mu)}-\lim s_{m n}=\overline{1}, \\
& S_{2, \gamma 1, \gamma^{2}} \bar{N}(\lambda, \mu)
\end{aligned}
$$

which is impossible.

Example 2. Define $\left(s_{m n}\right)$ as

$$
s_{m n}= \begin{cases}b & \text { if } m=k^{2}, n=l^{2}, \\ \overline{0} & \text { otherwise, }\end{cases}
$$

for $i, j \in \mathbb{N}$, where $b$ stands for a fixed fuzzy number. Let $\gamma_{1}=\gamma_{2}=1$, and let $u_{m}=1$ and $v_{n}=1 \forall m, n$. Let $\lambda_{k}=k$ and $\mu_{l}=l$. Suppose $b_{\alpha}^{+}$is the upper bound of the $\alpha$-cut. Then, for $\varepsilon>0,0 \leq \alpha \leq 1$ and $i, j \in \mathbb{N}$, one writes

$$
d\left(s_{m n}, \overline{0}\right)= \begin{cases}\sup _{0 \leq \alpha \leq 1}\left|b_{\alpha}^{+}\right| & \text {if } m=k^{2}, n=l^{2} \\ \overline{0} & \text { otherwise. }\end{cases}
$$

Thus,

$$
\begin{aligned}
& \lim _{k, l \rightarrow \infty} \frac{1}{U_{\lambda_{k}}^{\gamma_{1}} V_{\mu_{l}}^{\gamma_{2}}}\left|\left\{(m, n), m \leq U_{\lambda_{k}}, n \leq V_{\mu_{l}}: u_{m} v_{n} d\left(s_{m n}, \overline{0}\right) \geq \varepsilon\right\}\right| \\
& \quad \leq \lim _{k, l \rightarrow \infty} \frac{\sqrt[2]{k} \sqrt[2]{l}}{k l}=0 .
\end{aligned}
$$

Hence, $s_{m n} \longrightarrow S_{2, \gamma_{1}, \gamma_{2}}^{\hat{N}(\lambda, \mu)} \overline{0}$ but $\left(s_{m n}\right)$ is not convergent.

Theorem 2. Suppose $0<\gamma_{1}, \gamma_{2}, \eta_{1}, \eta_{2} \leq 1$ such that $\gamma_{1} \leq \eta_{1}$ and $\gamma_{2} \leq \eta_{2}$. Then, $S_{2, \gamma_{1}, \gamma_{2}}^{\bar{N}(\lambda, \mu)} \subseteq S_{2, \eta_{1}, \eta_{2}}^{\bar{N}(\lambda, \mu)}$.

Proof. Let $\varepsilon>0$ be given, and let $\gamma_{1}, \gamma_{2}, \eta_{1}, \eta_{2} \in(0,1]$. If $\gamma_{1}$ $\leq \eta_{1}$ and $\gamma_{2} \leq \eta_{2}$, then, for every $\varepsilon>0$, we can write

$$
\begin{aligned}
& \frac{1}{U_{\lambda_{k}}^{\gamma_{1}} V_{\mu_{l}}^{\gamma_{2}}}\left|\left\{(m, n), m \leq U_{\lambda_{k}}, n \leq V_{\mu_{l}}: u_{m} v_{n} d\left(s_{m n}, s_{0}\right) \geq \varepsilon\right\}\right|, \\
& \quad \leq \frac{1}{U_{\lambda_{k}}^{\eta_{1}} V_{\mu_{l}}^{\eta_{2}}}\left|\left\{(m, n), m \leq U_{\lambda_{k}}, n \leq V_{\mu_{l}}: u_{m} v_{n} d\left(s_{m n}, s_{0}\right) \geq \varepsilon\right\}\right|,
\end{aligned}
$$

which yields that $S_{2, \gamma_{1}, \gamma_{2}}^{\bar{N}(\lambda, \mu)} \subseteq S_{2, \eta_{1}, \eta_{2}}^{\bar{N}(\lambda, \mu)}$.

Theorem 3. Suppose two fuzzy double sequence $\left(s_{m n}\right)$ and $\left(t_{m n}\right)$ such that $S_{2, \gamma_{1}, \gamma_{2}}^{\bar{N}(\lambda, \mu)}-\lim s_{m n}=s_{0}$ and $S_{2, \gamma_{1}, \gamma_{2}}^{\bar{N}(\lambda, \mu)}-\lim t_{m n}=$ $t_{0}$. Then,

$$
\begin{aligned}
& \text { (i) } S_{2, \gamma_{1}, \gamma_{2}}^{\bar{N}(\lambda, \mu)}-\lim c s_{m n}=c s_{0}(c \in \mathbb{R}) \\
& \text { (ii) } S_{2, \gamma_{1}, \gamma_{2}}^{\bar{N}(\lambda, \mu)}-\lim \left(s_{m n}+t_{m n}\right)=s_{0}+t_{0}
\end{aligned}
$$

Proof.

(i) Let $S_{2, \gamma_{1}, \gamma_{2}}^{\bar{N}(\lambda, \mu)}-\lim s_{m n}=s_{0}$. It is clear for $c=0$. Let $c \neq 0$. For $\varepsilon>0$, we write

$$
\begin{aligned}
& \frac{1}{U_{\lambda_{k}}^{\gamma_{1}} V_{\mu_{l}}^{\gamma_{2}}}\left|\left\{(m, n), m \leq U_{\lambda_{k}}, n \leq V_{\mu_{l}}: u_{m} v_{n} d\left(c s_{m n}, c s_{0}\right) \geq \varepsilon\right\}\right| \\
& \quad=\frac{1}{U_{\lambda_{k}}^{\gamma_{1}} V_{\mu_{l}}^{\gamma_{2}}} \mid\left\{(m, n), m \leq U_{\lambda_{k}}, n\right. \\
& \left.\leq V_{\mu_{l}}: u_{m} v_{n}|c| d\left(s_{m n}, s_{0}\right) \geq \varepsilon\right\} \mid \\
& =\frac{1}{U_{\lambda_{k}}^{\gamma_{1}} V_{\mu_{l}}^{\gamma_{2}}} \mid\left\{(m, n), m \leq U_{\lambda_{k}}, n\right. \\
& \left.\leq V_{\mu_{l}}: u_{m} v_{n} d\left(s_{m n}, s_{0}\right) \geq \frac{\varepsilon}{|c|}\right\} \mid
\end{aligned}
$$

Thus, (i) holds. 
Suppose that $S_{2, \gamma_{1}, \gamma_{2}}^{\bar{N}(\lambda, \mu)}-\lim s_{m n}=s_{0}$ and $S_{2, \gamma_{1}, \gamma_{2}}^{\bar{N}(\lambda, \mu)}-\lim t_{m n}=$ $t_{0}$. Let $\varepsilon>0$ be given. Then,

$$
\begin{aligned}
& \frac{1}{U_{\lambda_{k}}^{\gamma_{1}} V_{\mu_{l}}^{\gamma_{2}}} \mid\left\{(m, n), m \leq U_{\lambda_{k}}, n \leq V_{\mu_{l}}: u_{m} v_{n} d\left(s_{m n}+t_{m n}, s_{0}+t_{0}\right)\right. \\
& \quad \geq \varepsilon\}\left|\leq \frac{1}{U_{\lambda_{k}}^{\gamma_{1}} V_{\mu_{l}}^{\gamma_{2}}}\right|\left\{(m, n), m \leq U_{\lambda_{k}}, n \leq V_{\mu_{l}}: u_{m} v_{n} d\left(s_{m n}, s_{0}\right)\right. \\
& \left.\quad \geq \frac{\varepsilon}{2}\right\}\left|+\frac{1}{U_{\lambda_{k}}^{\gamma_{1}} V_{\mu_{l}}^{\gamma_{2}}}\right|\left\{(m, n), m \leq U_{\lambda_{k}}, n\right. \\
& \left.\quad \leq V_{\mu_{l}}: u_{m} v_{n} d\left(t_{m n}, t_{0}\right) \geq \frac{\varepsilon}{2}\right\} \mid
\end{aligned}
$$

Hence, (ii) holds.

Definition 4. Assume that $\gamma_{1}, \gamma_{2} \in(0,1]$. Then, a fuzzy double sequence $\left(s_{m n}\right)$ is said to be strongly weighted $(\lambda, \mu)$-summable of order $\left(\gamma_{1}, \gamma_{2}\right)$; in short, we shall write $S_{2, \gamma_{1}, \gamma_{2}}^{\bar{N}(\lambda, \mu)}$-summable, to fuzzy number $s_{0}$, denoted by

$$
s_{m n} \stackrel{M_{2, \gamma_{1}, \gamma_{2}}^{N}}{\longrightarrow} s_{0}
$$

such that

$$
\lim _{k, l \rightarrow \infty} \frac{1}{U_{\lambda_{k}}^{\gamma_{1}} V_{\mu_{l}}^{\gamma_{2}}} \sum_{m \in\left[k-\lambda_{k}+1, k\right]} \sum_{n \in\left[l-\mu_{l}+1, l\right]} u_{m} v_{n} d\left(s_{m n}, s_{0}\right)=0 .
$$

Theorem 5. Consider a fuzzy double sequence $s=\left(s_{m n}\right)$, and let $0<\gamma_{1}, \gamma_{2} \leq 1$. Then,

(i) $s_{m n} \stackrel{M_{2, \gamma_{1}, \gamma_{2}}^{\bar{N}(\lambda, \mu)}}{\longrightarrow} s_{0}$ implies $s_{m n} \stackrel{S_{2, \gamma_{1}, \gamma_{2}}^{\bar{N}(\lambda, \mu)}}{\longrightarrow} s_{0}$

(ii) $\left(s_{m n}\right)$ is a bounded double sequence and $s_{m n} \stackrel{S_{2, \gamma_{1}, \gamma_{2}}^{\mathrm{N}(\lambda, \mu)}}{\longrightarrow} s_{0}$ imply $s_{m n} \stackrel{M_{2, \gamma_{1}, \gamma_{2}}^{\bar{N}(\lambda, \mu)}}{\longrightarrow} s_{0}$

Proof.

(i) Let $s_{m n} \stackrel{M_{2, \gamma_{1}, \gamma_{2}}^{N(\lambda, \mu)}}{\longrightarrow} s_{0}$, and let $\varepsilon>0$ be given. Then,

$$
\begin{gathered}
\sum_{m \in\left[k-\lambda_{k}+1, k\right] n \in\left[l-\mu_{l}+1, l\right]} \sum_{\substack{m \in\left[k-\lambda_{k}+1, k\right] \\
u_{m} v_{n} d\left(s_{m n}, s_{0}\right) \geq \varepsilon}} \sum_{\substack{n \in\left[l-\mu_{l}+1, l\right] \\
u_{m} v_{n} d\left(s_{m n}, s_{0}\right) \geq \varepsilon}} u_{m} v_{n} d\left(s_{m n}, s_{0}\right) \\
+\sum_{\substack{m \in\left[k-\lambda_{k}+1, k\right] \\
u_{m} v_{n} d\left(s_{m n}, s_{0}\right)<\varepsilon}} \sum_{\substack{n \in\left[l-\mu_{l}+1, l\right] \\
u_{m} v_{n} d\left(s_{m n}, s_{0}\right)<\varepsilon}} u_{m} v_{n} d\left(s_{m n}, s_{0}\right) \\
\geq \sum_{\substack{m \in\left[k-\lambda_{k}+1, k\right] \\
u_{m} v_{n} d\left(s_{m n}, s_{0}\right) \geq \varepsilon}} \sum_{\substack{n \in\left[l-\mu_{l}+1, l\right] \\
u_{m} v_{n} d\left(s_{m n}, s_{0}\right) \geq \varepsilon}} u_{m} v_{n} d\left(s_{m n}, s_{0}\right)
\end{gathered}
$$

Therefore, we get

$$
\begin{aligned}
& \frac{1}{U_{\lambda_{k}}^{\gamma_{1}} V_{\mu_{l}}^{\gamma_{2}}} \sum_{m \in\left[k-\lambda_{k}+1, k\right]} \sum_{n \in\left[l-\mu_{l}+1, l\right]} u_{m} v_{n} d\left(s_{m n}, s_{0}\right) \\
& \geq \frac{1}{U_{\lambda_{k}}^{\gamma_{1}} V_{\mu_{l}}^{\gamma_{2}}} \sum_{\substack{m \in\left[k-\lambda_{k}+1, k\right] \\
u_{m} v_{n} d\left(s_{m n}, s_{0}\right) \geq \varepsilon}} \sum_{\substack{n \in\left[l-\mu_{l}+1, l\right] \\
u_{m} v_{n} d\left(s_{m n}, s_{0}\right) \geq \varepsilon}} \varepsilon \\
& \geq \frac{1}{U_{\lambda_{k}}^{\gamma_{1}} V_{\mu_{l}}^{\gamma_{2}}} \mid\left\{(m, n), m \leq U_{\lambda_{k}}, n\right. \\
& \left.\leq V_{\mu_{l}}: u_{m} v_{n} d\left(s_{m n}, s_{0}\right) \geq \varepsilon\right\} \mid \varepsilon .
\end{aligned}
$$

Letting $k, l \longrightarrow \infty$ on both sides of the above relation, we get $s_{m n} \stackrel{S_{2, \gamma_{1}, \gamma_{2}}^{N}}{\longrightarrow} s_{0}$.

Let $s_{m n} \stackrel{s_{2, \gamma_{1}, \gamma_{2}}^{N(\lambda, \mu)}}{\longrightarrow} s_{0}$. Since $s=\left(s_{m n}\right) \in \mathscr{L}_{2, \infty}, d\left({ }_{m n}, s_{0}\right) \leq C$ for all $m, n \in \mathbb{N}$. For a given $\varepsilon>0$, one writes

$$
\begin{aligned}
& \frac{1}{U_{\lambda_{k}}^{\gamma_{1}} V_{\mu_{l}}^{\gamma_{2}}} \sum_{m \in\left[k-\lambda_{k}+1, k\right]} \sum_{n \in\left[l-\mu_{l}+1, l\right]} u_{m} v_{n} d\left(s_{m n}, s_{0}\right) \\
& =\frac{1}{U_{\lambda_{k}}^{\gamma_{1}} V_{\mu_{l}}^{\gamma_{2}}} \sum_{m \in\left[k-\lambda_{k}+1, k\right]} \sum_{n \in\left[l-\mu_{l}+1, l\right]} u_{m} v_{n} d\left(s_{m n}, s_{0}\right) \\
& u_{m} v_{n} d\left(s_{m n}, s_{0}\right) \geq \varepsilon u_{m} v_{n} d\left(s_{m n}, s_{0}\right) \geq \varepsilon \\
& +\frac{1}{U_{\lambda_{k}}^{\gamma_{1}} V_{\mu_{l}}^{\gamma_{2}}} \sum_{\substack{m \in\left[k-\lambda_{k}+1, k\right] \\
u_{m} v_{n} d\left(s_{m n}, s_{0}\right)<\varepsilon}} \sum_{\substack{n \in\left[l-\mu_{l}+1, l\right] \\
u_{m} v_{n} d\left(s_{m n}, s_{0}\right)<\varepsilon}} u_{m} v_{n} d\left(s_{m n}, s_{0}\right) \\
& \leq \frac{1}{U_{\lambda_{k}}^{\gamma_{1}} V_{\mu_{l}}^{\gamma_{2}}} \sum_{\substack{m \in\left[k-\lambda_{k}+1, k\right] \\
u_{m} v_{n} d\left(s_{m n}, s_{0}\right)<\varepsilon}} \sum_{\substack{n \in\left[l-\mu_{l}+1, l\right] \\
u_{m} v_{n} d\left(s_{m n}, s_{0}\right)<\varepsilon}} C \\
& +\frac{1}{U_{\lambda_{k}}^{\gamma_{1}} V_{\mu_{l}}^{\gamma_{2}}} \sum_{m \in\left[k-\lambda_{k}+1, k\right]} \sum_{n \in\left[l-\mu_{l}+1, l\right]} \varepsilon \\
& u_{m} v_{n} d\left(s_{m n}, s_{0}\right)<\varepsilon u_{m} v_{n} d\left(s_{m n}, s_{0}\right)<\varepsilon \\
& \leq \frac{1}{U_{\lambda_{k}}^{\gamma_{1}} V_{\mu_{l}}^{\gamma_{2}}} \mid\left\{(m, n), m \leq U_{\lambda_{k}}, n\right. \\
& \left.\leq V_{\mu_{l}}: u_{m} v_{n} d\left(s_{m n}, s_{0}\right) \geq \varepsilon\right\} \mid C+\varepsilon .
\end{aligned}
$$

$$
\text { It follows that } s_{m n} \stackrel{M_{2, \gamma_{1}, \gamma_{2}}^{\bar{N}(\lambda)}}{\longrightarrow} s_{0} \text {. }
$$

\section{Application to Fuzzy Korovkin- Type Theorems}

The fuzzy version of Korovkin theorem has been obtained by Anastassiou [34] while the classification of this result has been established by Korovkin in [35] (also see [36-42]), and then Anastassiou and Duman [43] and Karaisa and Kadak [44] studied this result in a statistical sense. In the recent past, Mohiuddine et al. [23] investigated the fuzzy Korovkin-type approximation theorem through weighted statistical convergence of fuzzy sequence based on difference operators. The fuzzy Korovkin theorem for function of two variables was discussed by Demirci and Karakus [45] with the help of $A$-statistical convergence for a sequence of fuzzy numbers. Here, we prove the fuzzy Korovkin-type theorem 
for the function of two variables with the help of $S_{2, \gamma_{1}, \gamma_{2}}^{\bar{N}(\lambda, \mu)}$ -convergence.

Assume that $I=[a, b](a, b \in \mathbb{R})$ and $g: I \times I\left(=I^{2}\right) \longrightarrow$ $\mathscr{L}(\mathbb{R})$ is a fuzzy number-valued function. Then, $g$ is fuzzy continuous at a point $S$ of $I \times I$ if $D(g(s), g(S)) \longrightarrow 0$ whenever $s=\left(s_{m n}\right)$ is Pringsheim's convergent to $S$ in a fuzzy sense. We denote the set of fuzzy continuous function on $I^{2}=[a, b]$ $\times[a, b]$ by $C_{\mathscr{L}}\left(I^{2}\right)$. Here, $C_{\mathscr{L}}\left(I^{2}\right)$ is only a cone but not a vector space and let $T: C_{\mathscr{L}}\left(I^{2}\right) \longrightarrow C_{\mathscr{L}}\left(I^{2}\right)$ be an operator. Further, $T$ is said to be a fuzzy linear operator if

$T\left(\beta_{1} \cdot g_{1} \oplus \beta_{2} \cdot g_{2} ; x, y\right)=\beta_{1} \cdot T\left(g_{1} ; x, y\right) \oplus \beta_{2} \cdot T\left(g_{2} ; x, y\right)$,

for all $\beta_{1}, \beta_{2} \in \mathbb{R}, g_{1}, g_{2} \in C_{\mathscr{L}}\left(I^{2}\right)$, and $(x, y) \in I^{2}$. In addition, if $T\left(g_{1} ; x, y\right)^{\circ} T\left(g_{2} ; x, y\right)$ holds for all $g_{1}, g_{2} \in C_{\mathscr{L}}\left(I^{2}\right)$ and $(x, y) \in I^{2}$ with $g_{1}(x, y)^{\circ} g_{2}(x, y)$, then $T$ is called fuzzy position linear operator.

Theorem 6. Let $\left(A_{n r}\right)_{n, r \in \mathbb{N}}$ be a double sequence such that $A_{n r}: C_{\mathscr{L}}\left(I^{2}\right) \longrightarrow C_{\mathscr{L}}\left(I^{2}\right)$. Let $\left(\tilde{A}_{n r}\right)_{n, r \in \mathbb{N}}$ be corresponding positive linear operators acting from $C\left(I^{2}\right)$ into itself having the relation

$$
\begin{aligned}
\left\{A_{n r}(g ; x, y)\right\}_{\alpha}^{ \pm} & =\tilde{A}_{n r}\left(g_{\alpha}^{ \pm} ; x, y\right)(\forall \alpha \in[0,1],(x, y) \\
& \left.\in I^{2}, g \in C_{\mathscr{L}}\left(I^{2}\right), n, r \in \mathbb{N}\right) .
\end{aligned}
$$

Assume further that

$$
S_{2, \gamma_{1}, \gamma_{2}}^{\bar{N}(\lambda, \mu)}-\lim \left\|\tilde{A}_{n r}\left(g_{i}\right)-g_{i}\right\|=0(i=0,1,2,3),
$$

where $g_{0}\left(t_{1}, t_{2}\right)=1, g_{1}\left(t_{1}, t_{2}\right)=t_{1}, g_{2}\left(t_{1}, t_{2}\right)=t_{2}$, and $g_{3}\left(t_{1}\right.$, $\left.t_{2}\right)=t_{1}+t_{2}$. Then,

$$
S_{2, \gamma_{1}, \gamma_{2}}^{\bar{N}(\lambda, \mu)}-\lim d^{*}\left(A_{n r}(g), g\right)=0,
$$

$$
\text { for all } n, r \in \mathbb{N} \text { and } g \in C_{\mathscr{L}}\left(I^{2}\right) \text {. }
$$

Proof. Suppose that (31) holds and $(n, r) \in \mathbb{N} \times \mathbb{N}$. Suppose also that $(x, y) \in I^{2}, g \in C_{\mathscr{L}}\left(I^{2}\right)$, and $\alpha \in[0,1]$. Since $g_{\alpha}^{ \pm} \in$ $C\left(I^{2}\right)$, it follows that, for a given $\varepsilon>0$, there exists $\delta>0$ such that for all $\left(t_{1}, t_{2}\right) \in I^{2}$, we have $\left|g_{\alpha}^{ \pm}\left(t_{1}, t_{2}\right)-g_{\alpha}^{ \pm}(x, y)\right|$ $<\varepsilon$ whenever $\left|t_{1}-x\right|<\delta$ and $\left|t_{2}-y\right|<\delta$.

By the fuzzy boundedness of $g$, we may write $\left|g_{\alpha}^{ \pm}(x, y)\right|$ $\leq C_{\alpha}^{ \pm}$for all $(x, y) \in I^{2}$, where $C_{\alpha}^{ \pm}=\left\|g_{\alpha}^{ \pm}\right\|$for all $a<y<b$. We then obtain for all $\left(t_{1}, t_{2}\right),(x, y) \in I^{2}$ that

$$
\left|g_{\alpha}^{ \pm}\left(t_{1}, t_{2}\right)-g_{\alpha}^{ \pm}(x, y)\right| \leq 2 C_{\alpha}^{ \pm} .
$$

With the help of last relations, for all $\left|t_{1}-x\right|<\delta$ and $\left|t_{2}-y\right|<\delta$, we obtain

$$
\left|g_{\alpha}^{ \pm}\left(t_{1}, t_{2}\right)-g_{\alpha}^{ \pm}(x, y)\right|<\varepsilon+\frac{2 C_{\alpha}^{ \pm}}{\delta^{2}}\left\{\left(t_{1}-x\right)^{2}+\left(t_{2}-y\right)^{2}\right\}
$$

Since $\tilde{A}_{n r}$ is a positive linear operator, by applying this operator to (34), we get

$$
\begin{aligned}
\mid \tilde{A}_{n r} & \left(g_{\alpha}^{ \pm}\left(t_{1}, t_{2}\right) ; x, y\right)-g_{\alpha}^{ \pm}(x, y) \mid \\
= & \mid \tilde{A}_{n r}\left(g_{\alpha}^{ \pm}\left(t_{1}, t_{2}\right)-g_{\alpha}^{ \pm}(x, y) ; x, y\right) \\
& +g_{\alpha}^{ \pm}(x, y)\left[\tilde{A}_{n r}\left(g_{0} ; x, y\right)-g_{0}(x, y)\right] \mid \\
\leq & \tilde{A}_{n r}\left(\left|g_{\alpha}^{ \pm}\left(t_{1}, t_{2}\right)-g_{\alpha}^{ \pm}(x, y)\right| ; x, y\right) \\
& +C_{\alpha}^{ \pm}\left|\tilde{A}_{n r}\left(g_{0} ; x, y\right)-g_{0}(x, y)\right| \\
\leq & \tilde{A}_{n r}\left(\varepsilon+\frac{2 C_{\alpha}^{ \pm}}{\delta^{2}}\left[\left(t_{1}-x\right)^{2}+\left(t_{2}-y\right)^{2}\right] ; x, y\right) \\
& +C_{\alpha}^{ \pm}\left|\tilde{A}_{n r}\left(g_{0} ; x, y\right)-g_{0}(x, y)\right| \\
\leq & +\left(\varepsilon+C_{\alpha}^{ \pm}+\frac{4 C_{\alpha}^{ \pm} E^{2}}{\delta^{2}}\right)\left|\tilde{A}_{n r}\left(g_{0} ; x, y\right)-g_{0}(x, y)\right| \\
& +\frac{4 C_{\alpha}^{ \pm} E}{\delta^{2}}\left|\tilde{A}_{n r}\left(g_{1} ; x, y\right)-g_{1}(x, y)\right| \\
& +\frac{4 C_{\alpha}^{ \pm} E}{\delta^{2}}\left|\tilde{A}_{n r}\left(g_{2} ; x, y\right)-g_{2}(x, y)\right| \\
& +\frac{2 C_{\alpha}^{ \pm}}{\delta^{2}}\left|\tilde{A}_{n r}\left(g_{3} ; x, y\right)-g_{3}(x, y)\right|,
\end{aligned}
$$

where $E=\max \{|x|,|y|\}$. We thus obtain by letting

$$
\Omega_{\alpha}^{ \pm}(\varepsilon)=\max \left\{\varepsilon+C_{\alpha}^{ \pm}+\frac{4 C_{\alpha}^{ \pm} E^{2}}{\delta^{2}}, \frac{4 C_{\alpha}^{ \pm} E}{\delta^{2}}, \frac{2 C_{\alpha}^{ \pm} E}{\delta^{2}}\right\}
$$

and taking $\sup _{(x, y) \in I^{2}}$ that

$$
\begin{aligned}
\left\|\tilde{A}_{n r}\left(g_{\alpha}^{ \pm}\right)-g_{\alpha}^{ \pm}\right\| \leq & \varepsilon+\Omega_{\alpha}^{ \pm}(\varepsilon)\left\{\left\|\tilde{A}_{n r}\left(g_{0}\right)-g_{0}\right\|\right. \\
& +\left\|\tilde{A}_{n r}\left(g_{1}\right)-g_{1}\right\|+\left\|\tilde{A}_{n r}\left(g_{2}\right)-g_{2}\right\| \\
& \left.+\left\|\tilde{A}_{n r}\left(g_{3}\right)-g_{3}\right\|\right\} .
\end{aligned}
$$

From (30), we can write

$$
\begin{aligned}
d^{*}\left(A_{m r}(g), g\right)= & \sup _{(x, y) \in I^{2}} d\left(A_{m r}(g ; x, y), g(x, y)\right) \\
= & \sup _{(x, y) \in I^{2} \alpha \in[0,1]} \sup _{\max } \max \left\{\left|\tilde{A}_{m r}\left(g_{\alpha}^{-} ; x, y\right)-g_{\alpha}^{-}(x, y)\right|,\right. \\
& \left.\left|\tilde{A}_{m r}\left(g_{\alpha}^{+} ; x, y\right)-g_{\alpha}^{+}(x, y)\right|\right\},
\end{aligned}
$$


and so

$$
\begin{aligned}
& d^{*}\left(A_{n r}(g), g\right) \\
& \quad=\sup _{\alpha \in[0,1]} \max \left\{\left\|\tilde{A}_{n r}\left(g_{\alpha}^{-}\right)-g_{\alpha}^{-}\right\|,\left\|\tilde{A}_{n r}\left(g_{\alpha}^{+}\right)-g_{\alpha}^{+}\right\|\right\} .
\end{aligned}
$$

With the help of (37) and (39), we get

$$
\begin{aligned}
d^{*}\left(A_{n r}(g), g\right) \leq & \varepsilon+\Omega(\varepsilon)\left\{\left\|\tilde{A}_{n r}\left(g_{0}\right)-g_{0}\right\|+\left\|\tilde{A}_{n r}\left(g_{1}\right)-g_{1}\right\|\right. \\
& \left.+\left\|\tilde{A}_{n r}\left(g_{2}\right)-g_{2}\right\|+\left\|\tilde{A}_{n r}\left(g_{3}\right)-g_{3}\right\|\right\},
\end{aligned}
$$

where

$$
\Omega(\varepsilon)=\sup _{\alpha \in[0,1]} \max \left\{\Omega_{\alpha}^{-}(\varepsilon), \Omega_{\alpha}^{+}(\varepsilon)\right\}
$$

Multiplying by the product of two nonnegative sequences $\left(u_{n}\right)$ and $\left(v_{r}\right)$ as details given in the previous section, one gets

$$
\begin{aligned}
u_{n} v_{r} d^{*}\left(A_{n r}(g), g\right) \leq & u_{n} v_{r} \varepsilon+\Omega(\varepsilon)\left\{u_{n} v_{r}\left\|\tilde{A}_{n r}\left(g_{0}\right)-g_{0}\right\|\right. \\
& +u_{n} v_{r}\left\|\tilde{A}_{n r}\left(g_{1}\right)-g_{1}\right\| \\
& +u_{n} v_{r}\left\|\tilde{A}_{n r}\left(g_{2}\right)-g_{2}\right\| \\
& \left.+u_{n} v_{r}\left\|\tilde{A}_{n r}\left(g_{3}\right)-g_{3}\right\|\right\}
\end{aligned}
$$

For a given $\varrho>0$, choose $\varepsilon>0$ such that $\varepsilon u_{n} v_{r}<\varrho$. Then, upon setting,

$$
\begin{aligned}
& F=\left\{(n, r): u_{n} v_{r} d^{*}\left(A_{n r}(g), g\right) \geq \varrho\right\}, \\
& F_{0}=\left\{(n, r): u_{n} v_{r}\left\|\tilde{A}_{n r}\left(g_{0}\right)-g_{0}\right\| \geq \frac{\varrho-\varepsilon u_{n} v_{r}}{4 \Omega(\varepsilon)}\right\}, \\
& F_{1}=\left\{(n, r): u_{n} v_{r}\left\|\tilde{A}_{n r}\left(g_{1}\right)-g_{1}\right\| \geq \frac{\varrho-\varepsilon u_{n} v_{r}}{4 \Omega(\varepsilon)}\right\}, \\
& F_{2}=\left\{(n, r): u_{n} v_{r}\left\|\tilde{A}_{n r}\left(g_{2}\right)-g_{2}\right\| \geq \frac{\varrho-\varepsilon u_{n} v_{r}}{4 \Omega(\varepsilon)}\right\}, \\
& F_{3}=\left\{(n, r): u_{n} v_{r}\left\|\tilde{A}_{n r}\left(g_{3}\right)-g_{3}\right\| \geq \frac{\varrho-\varepsilon u_{n} v_{r}}{4 \Omega(\varepsilon)}\right\} .
\end{aligned}
$$

It follows that

$$
F \subset F_{0} \cup F_{1} \cup F_{2} \cup F_{3}
$$

Consequently, by taking weighted $(\lambda, \mu)$ density of order $\left(\gamma_{1}, \gamma_{2}\right)$, we obtain

$$
\begin{aligned}
\delta_{2, \gamma_{1}, \gamma_{2}}^{\bar{N}(\lambda, \mu)}(F) \leq & \delta_{2, \gamma_{1}, \gamma_{2}}^{\bar{N}(\lambda, \mu)}\left(F_{0}\right)+\delta_{2, \gamma_{1}, \gamma_{2}}^{\bar{N}(\lambda, \mu)}\left(F_{1}\right) \\
& +\delta_{2, \gamma_{1}, \gamma_{2}}^{\bar{N}(\lambda, \mu)}\left(F_{2}\right)+\delta_{2, \gamma_{1}, \gamma_{2}}^{\bar{N}(\lambda, \mu)}\left(F_{3}\right) .
\end{aligned}
$$

Using the hypothesis (31), we conclude that

$$
S_{2, \gamma_{1}, \gamma_{2}}^{\bar{N}(\lambda, \mu)}-\lim d^{*}\left(A_{n r}(g), g\right)=0
$$

holds for all $(n, r) \in \mathbb{N} \times \mathbb{N}$ and $g \in C_{\mathscr{L}}\left(I^{2}\right)$.

Recall that, for any nonnegative integer $j$, the $q$-integer $[j]_{q}(q>0)$ is given by

$$
[j]_{q}= \begin{cases}\frac{1-q^{j}}{1-q} & \text { if } q \neq 1, \\ j & \text { if } q=1\end{cases}
$$

and the $q$-factorial $[j]_{q}$ ! by

$$
\begin{aligned}
{[j]_{q} ! } & =[j]_{q} ![j-1]_{q} ! \cdots[1]_{q} ! \\
{[0]_{q} ! } & =1 .
\end{aligned}
$$

The $q$-binomial coefficients, for $0 \leq m \leq j$, is defined by

$$
\left[\begin{array}{l}
j \\
m
\end{array}\right]_{q}=\frac{[j]_{q} !}{[m]_{q} ![j-m]_{q} !}
$$

The bivariate case of classical $q$-Bernstein operators were introduced and studied by Barbosu [46]. We are now considering the fuzzy analogue of these operators to construct an example to illustrate our last theorem.

Example 3. Consider the fuzzy analogue of bivariate $q$ -Bernstein operators, defined by

$$
\begin{aligned}
B_{n r}^{\mathscr{L}, q_{1}, q_{2}}(g ; x, y) & =\bigoplus_{k=0}^{n} \bigoplus_{m=0}^{r} g\left(\frac{[k]_{q_{1}}}{[n]_{q_{1}}}, \frac{[m]_{q_{2}}}{[r]_{q_{2}}}\right) \\
& \odot b_{k, m, n, r}^{q_{1}, q_{2}}(x, y)(n, r \in \mathbb{N}),
\end{aligned}
$$

for all $g \in C_{\mathscr{L}}\left(I^{2}\right)\left(I^{2}=[0,1] \times[0,1]\right), \quad(x, y) \in[0,1] \times[0,1]$, and $0<q_{1}, q_{2}<1$, where

$$
b_{k, m, n, r}^{q_{1}, q_{2}}(x, y)=\left[\begin{array}{l}
n \\
k
\end{array}\right]_{q_{1}}\left[\begin{array}{c}
r \\
m
\end{array}\right]_{q_{2}} x^{k}(1-x)_{q_{1}}^{n-k} y^{m}(1-y)_{q_{2}}^{r-m} .
$$

Note that

$$
(1-x)_{q}^{n}=\prod_{k=0}^{n-1}\left(1-q^{k} x\right)
$$


The above operators $B_{n r}^{\mathscr{L}, q_{1}, q_{2}}(g ; x, y)$ are positive and linear, and one writes

$$
\begin{aligned}
& \left\{B_{n r}^{\mathscr{L}, q_{1}, q_{2}}(g ; x, y)\right\}_{\alpha}^{ \pm} \\
& \quad=B_{n r}^{q_{1}, q_{2}}\left(g_{\alpha}^{ \pm} ; x, y\right) \\
& \quad=\sum_{k=0}^{n} \sum_{m=0}^{r} b_{k, m, n, r}^{q_{1}, q_{2}}(x, y) g_{\alpha}^{ \pm}\left(\frac{[k]_{q_{1}}}{[n]_{q_{1}}}, \frac{[m]_{q_{2}}}{[r]_{q_{2}}}\right),
\end{aligned}
$$

for all $\left.0 \leq \alpha \leq 1, g_{\alpha}^{ \pm} \in C[0,1]\right)$. Suppose that $q_{1}=q_{1 n}$ and $q_{2}=q_{2 r}$ such that $q_{1 n} \longrightarrow 1$ as $s \longrightarrow \infty$ and $q_{2 r} \longrightarrow 1$ as $r$ $\longrightarrow \infty$. In view of this assumption, one can easily find that

$$
\begin{aligned}
& {[n]_{q_{1}} \longrightarrow \infty(n \longrightarrow \infty),} \\
& {[r]_{q_{2}} \longrightarrow \infty(r \longrightarrow \infty) .}
\end{aligned}
$$

We define the sequence of positive linear operators by

$$
\tilde{\Phi}_{n r}\left(g_{\alpha}^{ \pm} ; x, y\right)=\left(1+s_{n r}\right) B_{n r}^{q_{1}, q_{2}}\left(g_{\alpha}^{ \pm} ; x, y\right)
$$

In this case, $\left(s_{n r}\right)$ is same as defined in Example 2 with $\gamma_{1}=\gamma_{2}=1, \lambda_{k}=k, \mu_{l}=l, u_{n}=1$, and $v_{r}=1$ for all $n, r \in \mathbb{N}$. Therefore, we get

$$
\begin{aligned}
& \tilde{\Phi}_{n r}\left(g_{0} ; x, y\right)=1+s_{n r}, \\
& \tilde{\Phi}_{n r}\left(g_{1} ; x, y\right)=\left(1+s_{n r}\right) x, \\
& \tilde{\Phi}_{n r}\left(g_{2} ; x, y\right)=\left(1+s_{n r}\right) y, \\
& \tilde{\Phi}_{n r}\left(g_{3} ; x, y\right)=\left(1+s_{n r}\right)\left(x^{2}+y^{2}+\frac{x-x^{2}}{[n]_{q_{1}}}+\frac{y-y^{2}}{[r]_{q_{2}}}\right) .
\end{aligned}
$$

Since

$$
S_{2, \gamma_{1}, \gamma_{2}}^{\bar{N}(\lambda, \mu)}-\lim s_{n r}=0
$$

we observe that

$$
S_{2, \gamma_{1}, \gamma_{2}}^{\bar{N}(\lambda, \mu)}-\lim \left\|\tilde{\Phi}_{n r}\left(g_{i}\right)-g_{i}\right\|=0(i=0,1,2,3)
$$

Consequently, by Theorem 6, we obtain

$$
S_{2, \gamma_{1}, \gamma_{2}}^{\bar{N}(\lambda, \mu)}-\lim d^{*}\left(\Phi_{n r}(g), g\right)=0
$$

for all $g \in C_{\mathscr{L}}\left(I^{2}\right)$. Hence, all the conditions of Theorem 6 hold true for our operators $\tilde{\Phi}_{n r}$ but Theorem 2.2 obtained in [45] for convergence in Pringsheim's sense does not work for $\tilde{\Phi}_{n r}$ since the fuzzy double sequence $\left(s_{n r}\right)$ is not convergent in Pringsheim's sense. Thus, we conclude that Theorem 6 is stronger than the one proved for convergence in Pringsheim's sense.

\section{Data Availability}

Not applicable.

\section{Conflicts of Interest}

The author declares there are no conflicts of interest.

\section{Acknowledgments}

This project was funded by the Deanship of Scientific Research (DSR) at King Abdulaziz University, Jeddah, under grant no. (RG-36-130-38). The author, therefore, acknowledges with thank DSR for technical and financial support.

\section{References}

[1] V. Karakaya and T. Chishti, "Weighted statistical convergence," Iranian Journal of Science and Technology. Transaction A, Science, vol. 33, pp. 219-223, 2009.

[2] H. Fast, "Sur la convergence statistique," Colloquium Mathematicum, vol. 2, pp. 241-244, 1949.

[3] M. Mursaleen, V. Karakaya, M. Erturk, and F. Gursoy, "Weighted statistical convergence and its application to Korovkin type approximation theorem," Applied Mathematics and Computation, vol. 218, no. 18, pp. 9132-9137, 2012.

[4] C. Belen and S. A. Mohiuddine, "Generalized weighted statistical convergence and application," Applied Mathematics and Computation, vol. 219, no. 18, pp. 9821-9826, 2013.

[5] S. Ghosal, "Generalized weighted random convergence in probability," Applied Mathematics and Computation, vol. 249, pp. 502-509, 2014.

[6] R. Çolak and Ç. A. Bektaş, " $\lambda$-Statistical convergence of order $\alpha$," Acta Mathematica Scientia, vol. 31, no. 3, pp. 953-959, 2011.

[7] U. Kadak and S. A. Mohiuddine, "Generalized statistically almost convergence based on the difference operator which includes the (p, q)-Gamma function and related approximation theorems," Results in Mathematics, vol. 73, no. 1, article 9, 2018.

[8] S. A. Mohiuddine, "Statistical weighted A-summability with application to Korovkin's type approximation theorem," Journal of Inequalities and Applications, vol. 2016, article 101, 2016.

[9] S. A. Mohiuddine and B. Hazarika, "Some classes of ideal convergent sequences and generalized difference matrix operator," Filomat, vol. 31, no. 6, pp. 1827-1834, 2017.

[10] M. Mursaleen and S. Mohiuddine, "On ideal convergence in probabilistic normed spaces," Mathematica Slovaca, vol. 62, no. 1, pp. 49-62, 2012.

[11] R. Çolak, Statistical convergence of order $\alpha$, Modern methods in analysis and its applications, Anamaya Pub., New Delhi, 2010.

[12] A. D. Gadjiev and C. Orhan, "Some approximation theorems via statistical convergence," The Rocky Mountain Journal of Mathematics, vol. 32, no. 1, pp. 129-138, 2002.

[13] J. S. Connor, "The statistical and strong $p$-Cesàro convergence of sequences," Analysis, vol. 8, pp. 47-63, 1988.

[14] A. Pringsheim, "Zur theorie der zweifach unendlichen Zahlenfolgen," Mathematische Annalen, vol. 53, no. 3, pp. 289-321, 1900. 
[15] M. Mursaleen and O. H. H. Edely, "Statistical convergence of double sequences," Journal of Mathematical Analysis and Applications, vol. 288, no. 1, pp. 223-231, 2003.

[16] R. Çolak and Y. Altin, "Statistical convergence of double sequences of order $\tilde{\alpha}$," Journal of Function Spaces and Applications, vol. 2013, article 682823, 5 pages, 2013.

[17] M. Mursaleen, C. Çakan, S. A. Mohiuddine, and E. Savaş, "Generalized statistical convergence and statistical core of double sequences," Acta Mathematica Sinica, English Series, vol. 26, no. 11, pp. 2131-2144, 2010.

[18] M. Mursaleen, " $\lambda$-Statistical convergence," Mathematica Slovaca, vol. 50, no. 1, pp. 111-115, 2000.

[19] M. Cinar and M. Et, "Generalized weighted statistical convergence of double sequences and applications," Filomat, vol. 30, no. 3, pp. 753-762, 2016.

[20] S. A. Mohiuddine, A. Alotaibi, and M. Mursaleen, "Statistical convergence of double sequences in locally solid Riesz spaces," Abstract and Applied Analysis, vol. 2012, Article ID 719729, 9 pages, 2012.

[21] S. A. Mohiuddine, B. Hazarika, and A. Alotaibi, "On statistical convergence of double sequences of fuzzy valued functions," Journal of Intelligent Fuzzy Systems, vol. 32, no. 6, pp. 43314342, 2017.

[22] B. Hazarika, A. Alotaibi, and S. A. Mohiuddine, "Statistical convergence in measure for double sequences of fuzzyvalued functions," Soft Computing, vol. 24, no. 9, pp. 66136622, 2020.

[23] S. A. Mohiuddine, A. Asiri, and B. Hazarika, "Weighted statistical convergence through difference operator of sequences of fuzzy numbers with application to fuzzy approximation theorems," International Journal of General Systems, vol. 48, no. 5, pp. 492-506, 2019.

[24] J. S. Kwon, "On statistical andp-Cesaro convergence of fuzzy numbers," The Korean Journal of Computational \& Applied Mathematics, vol. 7, no. 1, pp. 195-203, 2000.

[25] F. Nuray and E. Savas, "Statistical convergence of sequences of fuzzy real numbers," Mathematica Slovaca, vol. 45, no. 3, pp. 269-273, 1995.

[26] E. Savas and M. Mursaleen, "On statistically convergent double sequences of fuzzy numbers," Information Sciences, vol. 162, pp. 183-192, 2004.

[27] H. Altinok, Y. Altin, and M. Isik, "Statistical convergence and strong $p$-Cesàro summability of order $\beta$ in sequences of fuzzy numbers," Iranian Journal of Fuzzy Systems, vol. 9, no. 2, pp. 65-75, 2012.

[28] H. Altinok and M. Et, "Statistical convergence of order $(\beta, \gamma)$ for sequences of fuzzy numbers," Soft Computing, vol. 23, no. 15, pp. 6017-6022, 2019.

[29] M. Et, H. Altinok, and R. Colak, "On $\lambda$-statistical convergence of difference sequences of fuzzy numbers," Information Sciences, vol. 176, no. 15, pp. 2268-2278, 2006.

[30] A. Karakaş, Y. Altın, and H. Altınok, "On generalized statistical convergence of order $\beta$ of sequences of fuzzy numbers," Journal of Intelligent \& Fuzzy Systems, vol. 26, no. 4, pp. 1909-1917, 2014.

[31] P. D. Srivastava and S. Ojha, " $\lambda$-Statistical convergence of fuzzy numbers and fuzzy functions of order $\theta$," Soft Comput, vol. 18, no. 5, pp. 1027-1032, 2014.

[32] L. A. Zadeh, "Fuzzy sets," Information and Control, vol. 8, no. 3, pp. 338-353, 1965.
[33] M. L. Puri and D. A. Ralescu, "Differentials of fuzzy functions," Journal of Mathematical Analysis and Applications, vol. 91, no. 2, pp. 552-558, 1983.

[34] G. A. Anastassiou, "On basic fuzzy Korovkin theory," Studia Universitatis Babeş-Bolyai Mathematica, vol. 50, pp. 3-10, 2005.

[35] P. P. Korovkin, Linear Operators and Approximation Theory, Hindustan Publ. Corp, Delhi, 1960.

[36] N. L. Braha, H. M. Srivastava, and S. A. Mohiuddine, "A Korovkin's type approximation theorem for periodic functions via the statistical summability of the generalized de la Vallée Poussin mean," Applied Mathematics and Computation, vol. 228, pp. 162-169, 2014.

[37] O. H. H. Edely, S. A. Mohiuddine, and A. K. Noman, "Korovkin type approximation theorems obtained through generalized statistical convergence," Applied Mathematics Letters, vol. 23, no. 11, pp. 1382-1387, 2010.

[38] B. B. Jena, S. K. Paikray, S. A. Mohiuddine, and V. N. Mishra, "Relatively equi-statistical convergence via deferred Nörlund mean based on difference operator of fractional-order and related approximation theorems," AIMS Mathematics, vol. 5, no. 1, pp. 650-672, 2020.

[39] S. A. Mohiuddine, "An application of almost convergence in approximation theorems," Applied Mathematics Letters, vol. 24, no. 11, pp. 1856-1860, 2011.

[40] S. A. Mohiuddine and B. A. S. Alamri, "Generalization of equistatistical convergence via weighted lacunary sequence with associated Korovkin and Voronovskaya type approximation theorems," Revista de la Real Academia de Ciencias Exactas, Físicas y Naturales. Serie A. Matemáticas, vol. 113, no. 3, pp. 1955-1973, 2019.

[41] S. A. Mohiuddine, B. Hazarika, and M. A. Alghamdi, "Ideal relatively uniform convergence with Korovkin and Voronovskaya types approximation theorems," Filomat, vol. 33, no. 14, pp. 4549-4560, 2019.

[42] H. M. Srivastava, B. B. Jena, S. K. Paikray, and U. K. Misra, "A certain class of weighted statistical convergence and associated Korovkin-type approximation theorems involving trigonometric functions," Mathematical Methods in the Applied Sciences, vol. 41, pp. 671-683, 2018.

[43] G. A. Anastassiou and O. Duman, "Statistical fuzzy approximation by fuzzy positive linear operators," Computers \& Mathematcs with Applications, vol. 55, no. 3, pp. 573-580, 2008.

[44] A. Karaisa and U. Kadak, "On $\alpha \beta$-statistical convergence for sequences of fuzzy mappings and Korovkin type approximation theorem," Filomat, vol. 31, no. 12, pp. 3749-3760, 2017.

[45] K. Demirci and S. Karakuş, "Four-dimensional matrix transformation and $A$-statistical fuzzy Korovkin type approximation," Demonstratio Mathematica, vol. 46, no. 1, pp. 37-49, 2013.

[46] D. Bărbosu, "Some generalized bivariate bernstein operators," Miskolc Mathematical Notes, vol. 1, no. 1, pp. 3-10, 2000. 